\title{
The effect of thermal activation on dislocation processes at an atomistic crack tip
}

\section{Y W Zhang, T C Wang and Q H Tang}

\author{
LNM, Institute of Mechanics, CAS, 100080, People's Republic of China
}

Received 20 December 1994

\begin{abstract}
The effects of thermal activation on the dislocation emission from an atomistic crack tip are discussed. Molecular dynamics simulations at different constant temperatures are carried out to investigate the thermal effects. The simulated results show that the processes of the partial dislocation generation and emission are temperature dependent. As the temperature increases, the incipient duration of the partial dislocation nucleation becomes longer, the critical stress intensity factor for partial dislocation emission is reduced and, at the same loading level, more dislocations are emitted. The dislocation velocity moving away from the crack tip and the separations of partial dislocations are apparently not temperature dependent. The simulated results also show that, as the temperature increases, the stress distribution along the crack increases slightly. Therefore stress softening at the crack tip induced by thermal activation does not exist in the present simulation. A simple model is proposed to evaluate the relation of the critical stress intensity factor versus temperature. The obtained relation is in good agreement with our molecular dynamics results.
\end{abstract}

\section{Introduction}

In the study of crack tip processes, both elasticity methods and atomistic methods were used to investigate the nucleation and emission of dislocations. However, the effects of thermal activation on the processes of dislocations were not considered completely. In fact, the temperature dependent effects have great influence on the fracture processes through the thermally activated process. The experimental results of Gilman [1] and Hirsch et al [2] concerning the temperature dependence of the fractal behaviour of crystals showed that there is a transition temperature, below which the fracture is brittle and above which the fracture is ductile.

In the work of Rice [3] and Rice and Beltz [4], $2 \mathrm{D}$ and $3 \mathrm{D}$ analyses have been given for the activation energy. They have found that thermal activation would be sufficient at room temperature to spontaneously emit a partial dislocation in copper at loading of the energy release rate $G=(0.2-0.3) G_{\text {crit }}$.

A model for the brittle-to-ductile transition considering both stress and temperature has been proposed by Khantha et al [5]. The temperature corresponding to a rapid increase in the free dislocation density, i.e. the brittle-to-ductile transition, is related to the stress level and the screening due to all dislocation dipoles.

An activation analysis of the nucleation of a half-square dislocation loop from a crack tip has been carried out by Cheung et al [6]. Their results show that the effects of temperature reduce the activation barrier for dislocation nucleation.

The molecular dynamics simulation using the Morse potential carried out by Mullins [7] shows that at low temperature the crack propagates as a brittle cleavage crack, while at high temperature the crack is blunted by the emitting dislocations and the transition is not sharp.

A discussion on the temperature dependence of the dislocation nucleation condition using the molecular dynamics method has also been given by Kitagawa and Nakatani [8]. They found that the relation between thermally activated energy and temperature is linear, and the relation between the critical stress intensity factor for dislocation emission and temperature is to the second power.

In the present paper we discuss the effects of temperature on the behaviour of dislocation emission from a crack tip. A molecular dynamics method is used to evaluate the temperature dependence. Several phenomena of dislocation processes at the atomistic crack tip related to the temperature are observed. Based on the works of Khantha et al [5], Kitagawa and Nakatani [8] and Li [9], a simple model is proposed to evaluate the effect of temperature on the critical condition for dislocation emission. An exponential relation between the critical stress intensity factor and temperature is obtained. This relation is in good agreement with our molecular dynamics results. 
along $\mathrm{x}$ : 90 periods, 180 layers

along y: 1 periods 6 layers

along z: 20 periods 101 layers

displacement boundaries

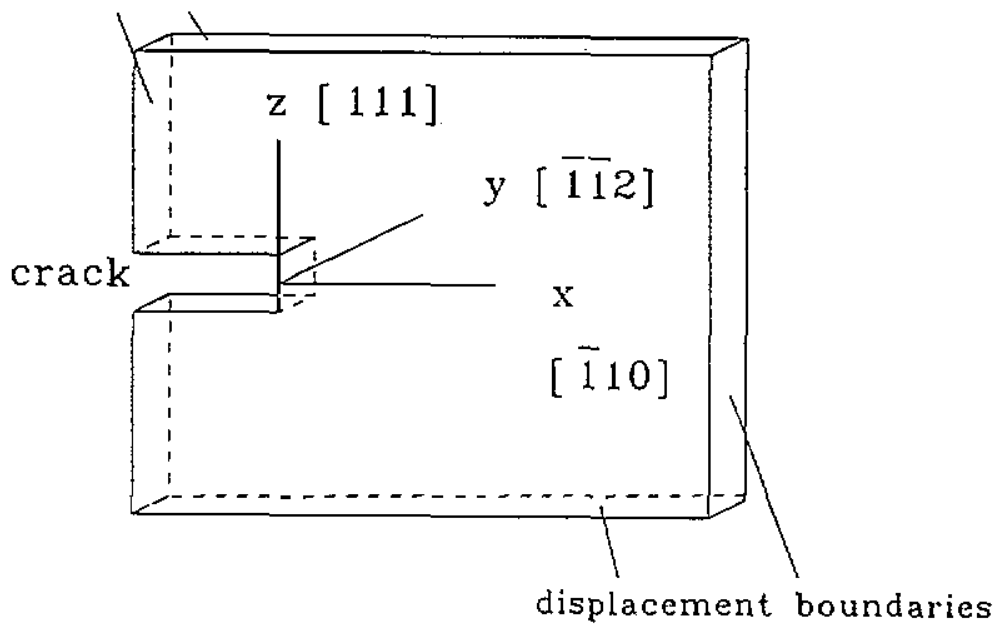

Figure 1. Crack tip and crystallographic geometry in an Fcc crystal.

\section{Atomic model for molecular dynamics simulation}

The molecular dynamics simulation method used in the present paper is the same as used by Zhang et al [10]. Here we illustrate the method briefly.

\subsection{Interatomic potential}

The interatomic potential used here is the ' $N$-body' potential proposed by Finnis and Sinclair [11] and constructed by Ackland et al [12]. The ansätz they used is

$$
U_{\mathrm{tot}}=-\sum_{i} \rho_{i}^{1 / 2}+\frac{1}{2} \sum_{i} \sum_{j(i \neq j)} V_{i j}
$$

where $\rho$ is the second moment of the density of states, and

$$
\rho_{i}=\sum_{j(i \neq j)} \Phi_{i j}
$$

where $V_{i j}$ and $\Phi_{i j}$ are functions only of the interatomic distance, and can be obtained by assuming some function forms and then fitting to the experimental data.

\subsection{Method of solution}

The mode II anisotropic elastic displacement field is used to prescribe the displacement of the border discrete atoms. The loading rate $K_{I I}$ is used as the loading controlled parameter. The inner atoms follow the laws of Newton

$$
\boldsymbol{F}_{i}=-\frac{\partial U_{\mathrm{tot}}}{\partial \boldsymbol{r}_{i}}=m_{i} a_{i}
$$

In the present paper, the leapfrog algorithm is used, which provides an update formulation. The time step in present calculation is taken to be $1.18 \times 10^{-14} \mathrm{~s}$. In all the simulations, the temperature of the systems is maintained at fixed values throughout the duration of the runs. The initial velocity is the Maxwell-Boltzmann distribution corresponding to a given temperature. An algorithm to maintain the temperature to a constant value proposed by Woodcock [13] and illustrated by Nose [14] is used to scale the temperature.

\subsection{The atom lattice geometry}

The $\{110\},\{111\}$ and $\{112\}$ crystallographic planes of the parallelepiped with a slit are used in the present calculations. The coordinate system is selected to be $x$, $y$ and $z$ axes along $\langle 110\rangle,\langle 112\rangle$ and $\langle 111\rangle$ directions respectively. In the FCC crystal, a full dislocation moves in the $\langle 110\rangle$ direction in the $\{111\}$ plane. So, in the present model, as shown in figure 1, the crack plane is taken to be in the $\{111\}$ plane, and the crack front is along the $\langle 112\}$ direction. The atom arrangement near the crack tip is given in figure 2. Under mode II loading, the dislocations move along the $\langle 110\rangle$ direction. The periodicity along $\langle 111\rangle$ is in three layers, along $\langle 110\rangle$ two layers and along $\langle 112\rangle$ six layers. Since a long extension in the slip direction is particularly important, the $\langle 110\rangle$ slip direction is made as extensive as is computationablly feasible.

A full dislocation in copper is generally dissociated into two partial dislocations. Between the two partial dislocations there is a faulted plane. As the periodicity condition is used along the (112) direction, the nucleation and motion of partial dislocations can be described with the present lattice configuration. 


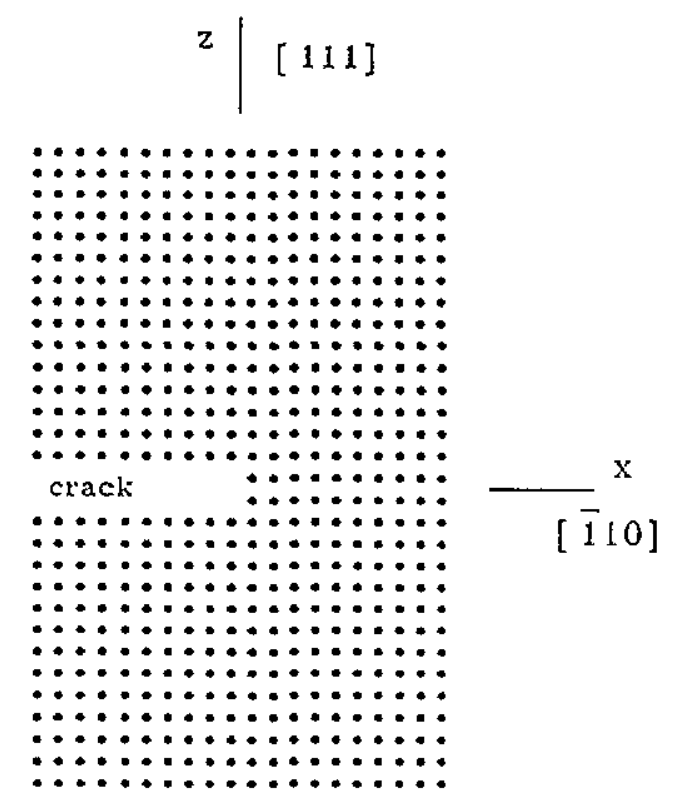

Figure 2. Atom arrangement near crack tip.

\subsection{The boundary conditions}

The boundary conditions applied to the boundary of the discrete atom region in our molecular dynamics simulation have been that of a prescribed displacement distribution dictated by a mode II anisotropic $K$ field in the $x-z$ plane. Along the $y$-direction, a six layer periodic representation is applied. Therefore the present atom lattice is actually three dimensional. The total number of atoms for the simulation is $N=18282$. The length along the $x$ direction is $(\sqrt{2} / 4) \times 180 a_{0}\left(a_{0}\right.$ is the lattice constant) and the width along the $z$-direction is $(\sqrt{3} / 3) \times 101 a_{0}$. The distance between the crack tip and the left boundary is $(\sqrt{2} / 4) \times 45 a_{0}$. As the length from the crack tip to the right boundary is large enough, the effect of the boundary constraints on the nucleation and emission of dislocations can be neglected if the dislocations are far from the boundary. On the other hand, if we take the boundary as an obstacle to block the moving of dislocations, then a pile-up of dislocations can then be set up.

\section{Results and discussion}

\subsection{Simulated results}

In the present paper, all the simulations are carried out with the variation of temperature. The loading rate is $K_{\mathrm{II}}=0.05085 \mathrm{MPa} \mathrm{m}^{1 / 2} \mathrm{ps}^{-1}$ and the total loading stress intensity factor is $0.6 \mathrm{MPa} \mathrm{m} \mathrm{m}^{1 / 2}$.

For the case of $T=0 \mathrm{~K}$, the curves of dislocation position versus loading level are given in figure 3 . There are five partial dislocations emitted. The first partial dislocation is emitted at $K_{\mathrm{II}}=0.2376 \mathrm{MPa} \mathrm{m}^{1 / 2}$. Once the dislocation has been formed, it only takes very short time for the partial dislocation to be accelerated to an approximate constant speed of $v=1442 \mathrm{~m} \mathrm{~s}^{-1}$. At $K_{\mathrm{I}}=0.295 \mathrm{MPa} \mathrm{m}{ }^{1 / 2}$, the second partial dislocation is emitted, it accelerates very quickly to the speed of the first dislocation. It can be seen that for copper crystal, the dislocation always takes the form of a partial one. The separation between a pair of partial dislocations of a full dislocation is shorter than the separation of a pair of partial dislocations belonging to different full dislocations.

For the case of $T=100 \mathrm{~K}$, the curves of dislocation position versus loading level are given in figure 4 . There are also five partial dislocations emitted. All critical values of the dislocation emission are reduced compared with the corresponding dislocation emission at $0 \mathrm{~K}$. The first partial dislocation is emitted at $K_{\mathrm{II}}=0.2154 \mathrm{MPa} \mathrm{m}^{1 / 2}$. The second partial dislocation is nucleated at $0.252 \mathrm{MPa}$ $\mathrm{m}^{1 / 2}$, then it returns to the crack tip. The second partial dislocation is not emitted until $K_{\mathrm{II}}=0.270 \mathrm{MPa} \mathrm{m}^{1 / 2}$. The curves are not as smooth as that of $T=0 \mathrm{~K}$.

For the case of $T=200 \mathrm{~K}$, the curves of dislocation position versus loading level are given in figure 5. There are six partial dislocations emitted. The critical values of the dislocation emission are reduced compared with the corresponding dislocation emission at $100 \mathrm{~K}$. The first partial dislocation is emitted at $K_{\mathrm{II}}=0.186 \mathrm{MPa} \mathrm{m}^{1 / 2}$. As the first partial dislocation reaches the displacement boundary, a pile-up of dislocations is set up. The curves shown in figure 5 becomes even more unsmooth.

For the case of $T=300 \mathrm{~K}$, the curves of dislocation position versus loading level are given in figure 6 . There are also six partial dislocations emitted. The dislocation has been nucleated at the crack tip many times, but it is not emitted from the tip. The formation of a dislocation does not mean it can be emitted. The incipient duration is the time between the first formation of dislocation and its emission. It can be seen that the incipient duration of the first partial dislocation of a full dislocation is longer than that of the second partial one. Due to thermal activation, the curves become even more unsmooth. The critical values of the dislocation emission are reduced compared with the corresponding dislocation emission at $200 \mathrm{~K}$. The first partial dislocation is emitted at $K_{\mathrm{II}}=0.165 \mathrm{MPa} \mathrm{m}{ }^{1 / 2}$. As the first partial dislocation reaches the displacement boundary, a pile-up of dislocations is also set up.

For the case of $T=400 \mathrm{~K}$, the curves of dislocation position versus loading level are given in figure 7. For this case there are also six partial dislocations emitted. The incipient duration of the first partial dislocation of a full dislocation becomes even longer than that of $T=300 \mathrm{~K}$. The first dislocation is nucleated at $K_{\mathrm{II}}=0.112 \mathrm{MPa} \mathrm{m} \mathrm{m}^{1 / 2}$ and emitted at $K_{\mathrm{I}}=0.146 \mathrm{MPa} \mathrm{m}^{1 / 2}$. After its emission, it does not move quickly away from the crack tip, but still hovers around the crack tip. The dislocation does not move away from the crack tip until $K_{\mathrm{n}}=0.167 \mathrm{MPa} \mathrm{m}^{1 / 2}$. Some partial dislocations are initially emitted from the crack tip, then return to the crack tip. As the loading continues to increase, the dislocations are re-emitted and driven away from the crack tip.

\subsection{Discussion}

From our simulated results it can be seen that the processes of the dislocation emission from the crack tip is temperature dependent. Through thermal activation, the critical stress intensity factors are reduced, dislocations can 


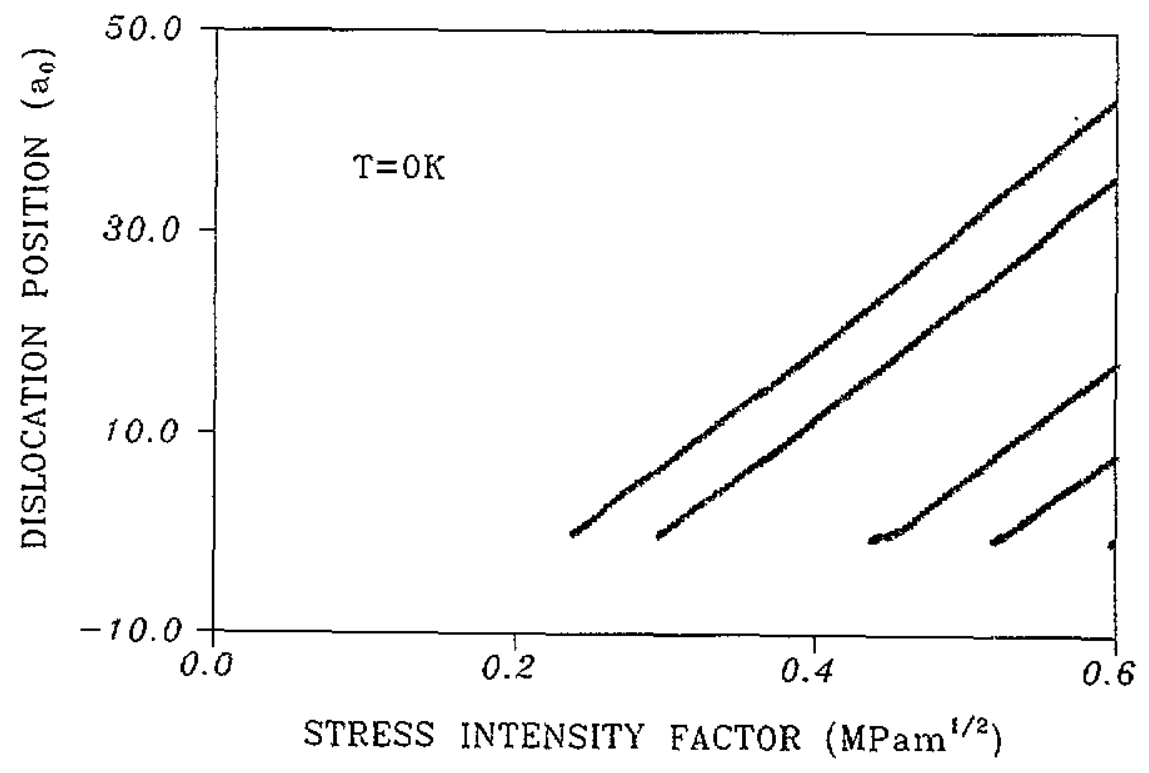

Figure 3. The variations of positions of the partial dislocations with loading level $K_{11}$ at temperature $0 \mathrm{~K}$. Five partial dislocations have been emitted.

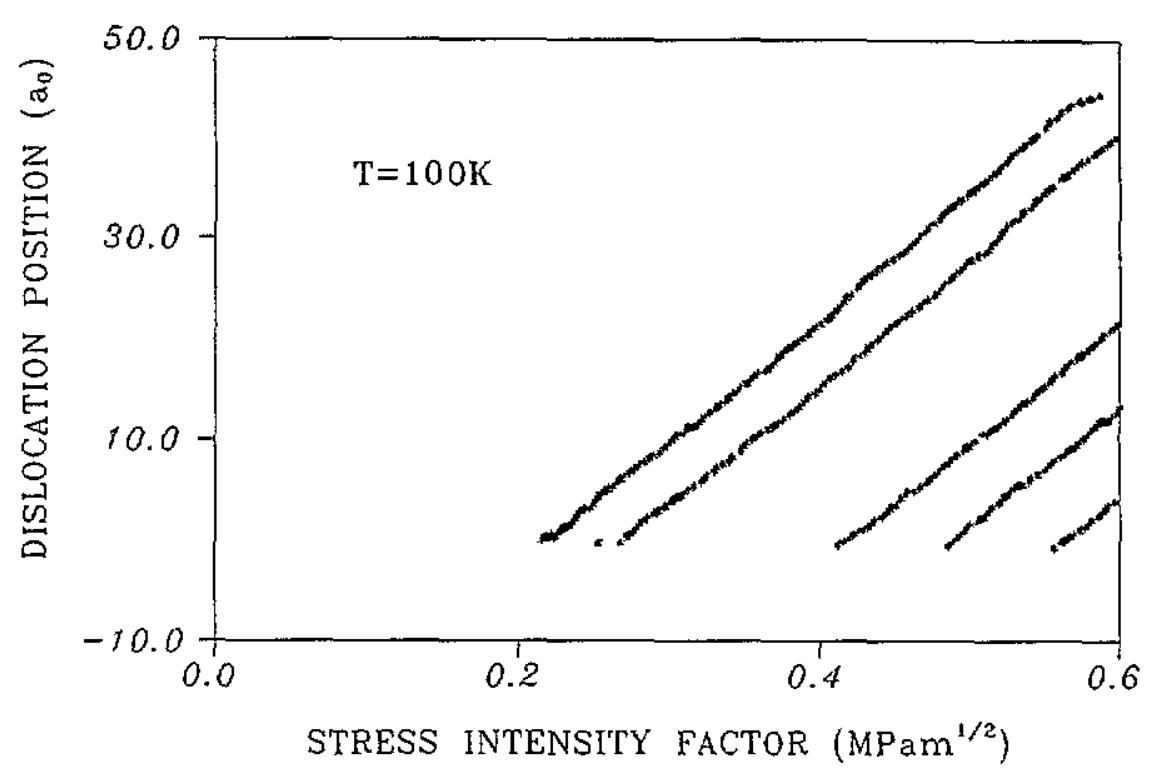

Figure 4. The variations of positions of the partial dislocations with loading level $K_{\| I}$ at temperature $100 \mathrm{~K}$. Five partial dislocations have been emitted.

be emitted more easily, and at the same loading level, more dislocations are emitted. At high temperatures, the dislocation velocity becomes unsmooth, the dislocations may move back to crack tip locally and instantaneously. There are also some behaviours which do not seem to exhibit temperature dependence. From $0-400 \mathrm{~K}$, the velocity of dislocations moving away from the crack tip increases slightly. The extended width of a pair of partial dislocations and the separation of a pair of partial dislocations belonging to two full dislocations exhibit an insensitivity to temperature. The stress distributions along the prolongation of crack tip at temperatures 0,77 and $200 \mathrm{~K}$ under $K_{\text {II }}=0.12 \mathrm{MPa} \mathrm{m}^{1 / 2}$ are plotted in figure 8 together with the anisotropic elasticity solution [15]. Due to the thermal fluctuation, the stress is evaluated through the average with both time and spatial location. It can be seen that the stress at $0 \mathrm{~K}$ is in good agreement with the anisotropic elasticity solution, except very near the crack tip. As the temperature increases, the stress level near crack tip increases slightly. Stress softening induced by thermal activation does not therefore exist in the present simulation.

\section{Temperature dependence of dislocation emission}

Generally, dislocation emission from a crack tip is a stress and temperature dependent process. At temperature $0 \mathrm{~K}$, the potential energy of a dislocation around the crack tip at 
$Y W$ Zhang et al

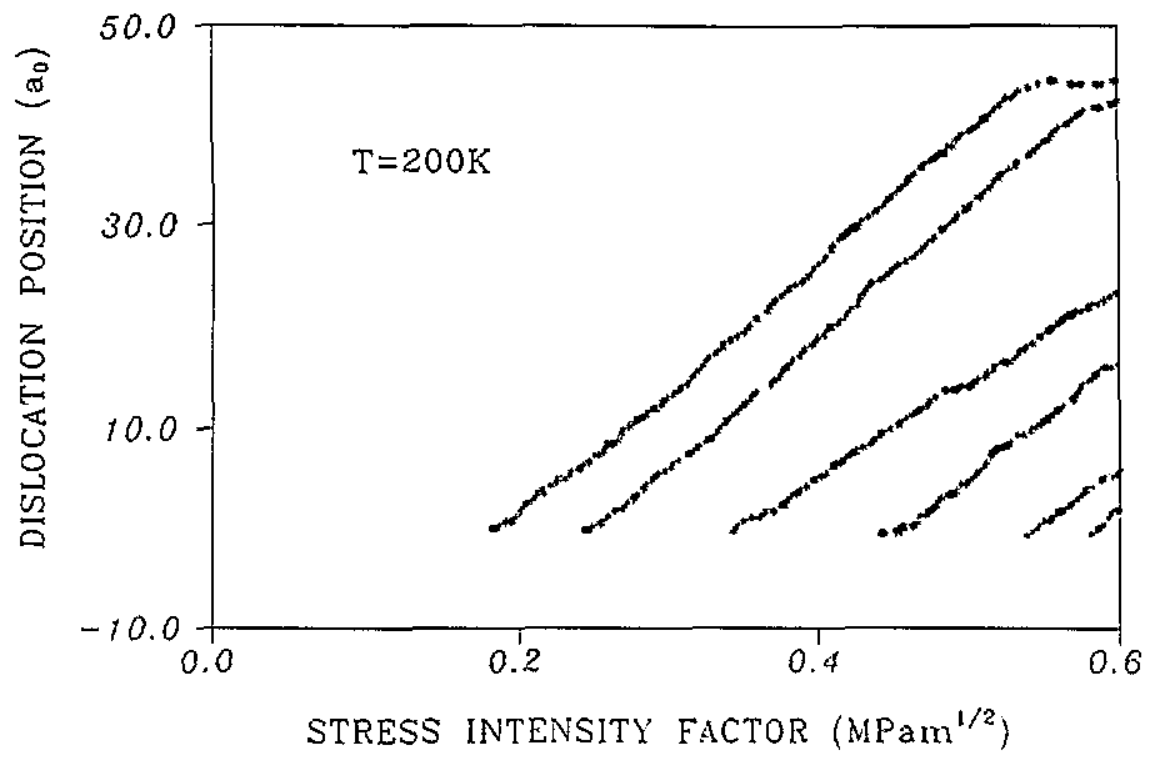

Figure 5. The variations of positions of the partial dislocations with loading level $K_{\|}$at temperature $200 \mathrm{~K}$. Six partial dislocations have been emitted.

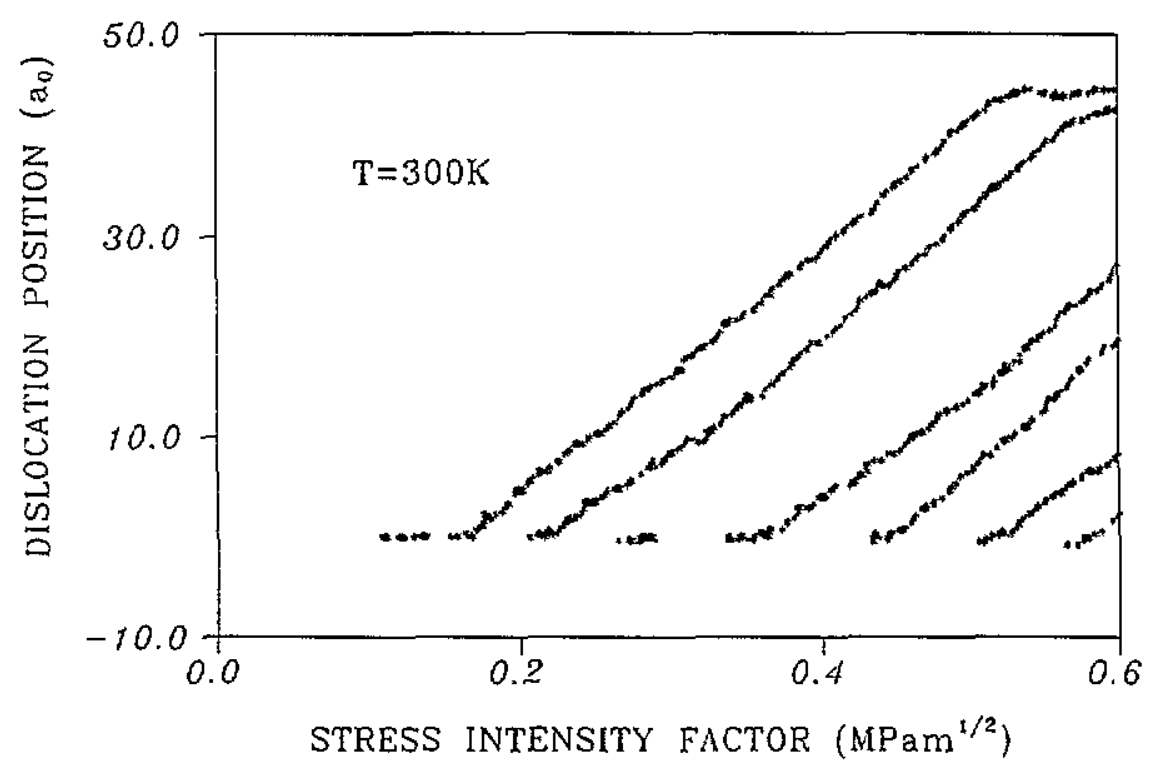

Figure 6. The variations of positions of the partial dislocations with loading level $K_{\mathrm{Il}}$ at temperature $300 \mathrm{~K}$. Six partial dislocations have been emitted.

the maximum was given by $\mathrm{Li}[9]$, i.e.

$$
\Delta G=A b^{2}\left(\ln \frac{A b}{K_{\mathrm{I}}^{0}} \sqrt{\frac{2 \pi}{r_{0}}}-1\right) .
$$

This leads to the critical stress intensity factor for dislocation emission at $0 \mathrm{~K}$ :

$$
K_{\mathrm{Ll} e}^{0}=\frac{A b}{e} \sqrt{\frac{2 \pi}{r_{0}}}
$$

where $e$ is the base for natural logarithm, $I_{0}$ is the core cutoff of dislocation, $A$ is $\mu / 2 \pi(1-\nu)$ for edge dislocation, $\mu$ is the shear modulus, $v$ is Poisson's ratio and $b$ is Burger's vector of the dislocation.
Since the dislocation emission process is a thermally activated process, the critical condition for dislocation emission will be reduced by the activation. In the works of Khantha et al [5] and Kitagawa and Nakatani [8], the thermal activation energy contributed by the temperature has a linear relation with temperature. Hence in the present paper we assume that the relation between activated energy and temperature $T$ is given by

$$
\Delta G_{T}=\frac{2 \alpha k_{\mathrm{B}} T}{b}
$$

per unit length of the dislocation, where $\alpha$ is the parameter related with material and loading rate and $k_{\mathrm{B}}$ is Boltzmann's constant. We also assume that the inherent activation of a dislocation is independent of temperature. So the critical 


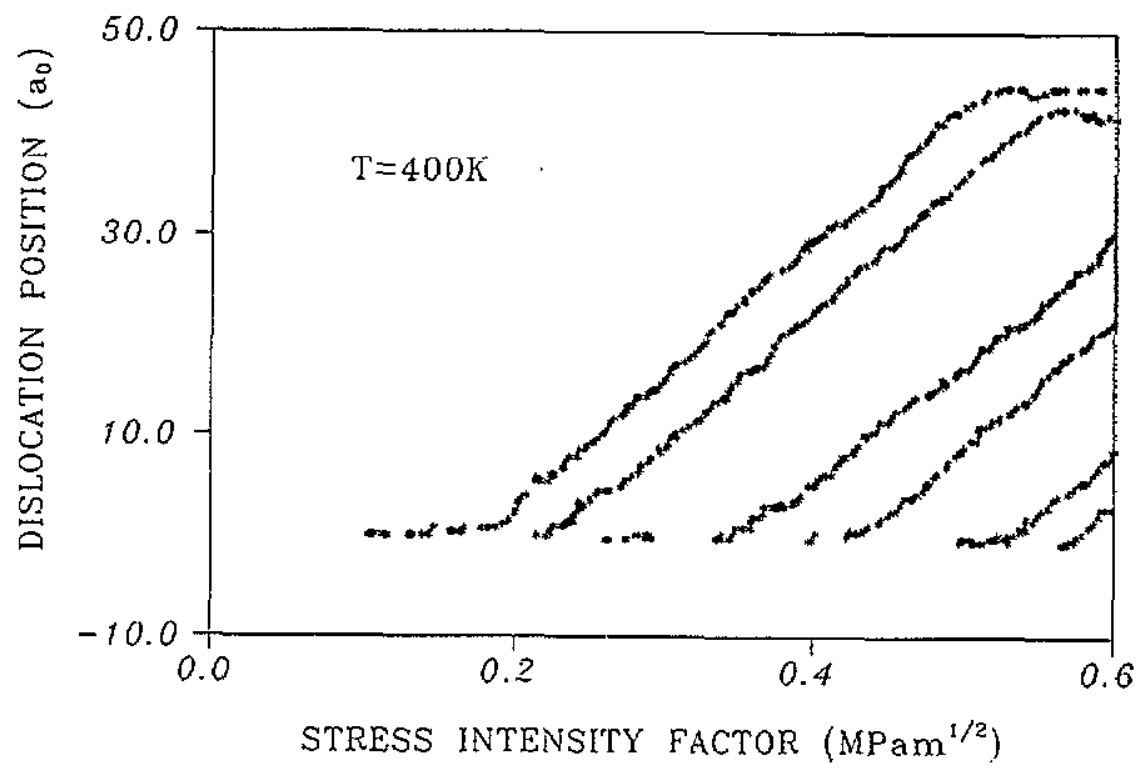

Figure 7. The variations of positions of the partial dislocations with loading level $K_{\| 1}$ at temperature $400 \mathrm{~K}$. Six partial dislocations have been emitted.

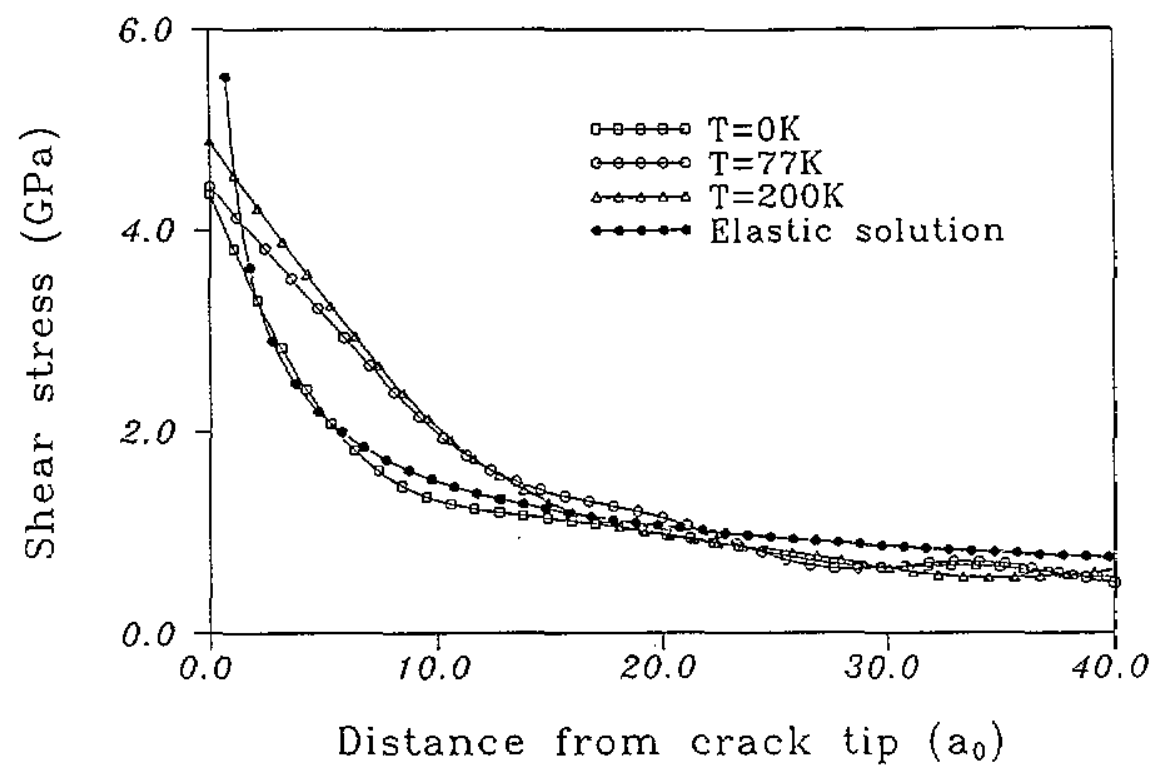

Figure 8. The simulated stress distributions along the prolongation of the crack at $T=0,77,200 \mathrm{~K}$, together with the anisotropic elasticity solution under $K_{\mathrm{ll}}=0.12 \mathrm{MPa} \mathrm{m}^{1 / 2}$.

condition for the dislocation emission becomes:

$$
A b^{2}\left[\ln \left(\frac{A b}{K_{\mathrm{I} e}} \sqrt{\frac{2 \pi}{r_{0}}}\right)-1\right]-\frac{2 \alpha k_{\mathrm{B}} T}{b}=0 .
$$

Thus we can obtain the critical condition for dislocation emission at temperature $T$ :

$$
K_{\mathbb{\Pi l} e}=K_{\mathrm{Me} e}^{0} \exp \left(-\frac{2 \alpha k_{\mathrm{B}} T}{A b^{3}}\right) .
$$

For copper, by using equation (5), we can obtain $K_{\text {Ile }}^{0}=0.15 \mathrm{MPa} \mathrm{m}{ }^{1 / 2}$, which is smaller than our molecular dynamics result of $0.2376 \mathrm{MPa} \mathrm{m}{ }^{1 / 2}$. If we let $K_{\text {II } e}^{0}=$ $0.2376 \mathrm{MPa} \mathrm{m}^{1 / 2}$ and $\alpha=0.9$, the result of equation (8) and the molecular dynamics result are shown in figure 9. It can be seen that they are in good agreement.

\section{Summary and conclusions}

The present molecular dynamics simulation of crack tip processes can give us several insights into and comprehensive understanding of nucleation and the emission of dislocations in relation to temperature. For copper, a full dislocation always dissociates into two partial dislocations. The extended width of a pair of two partial dislocations and the separation between two full dislocations are insensitive to thermal activation. The critical stress intensity factor for the partial dislocations $K_{\text {II }}$ 


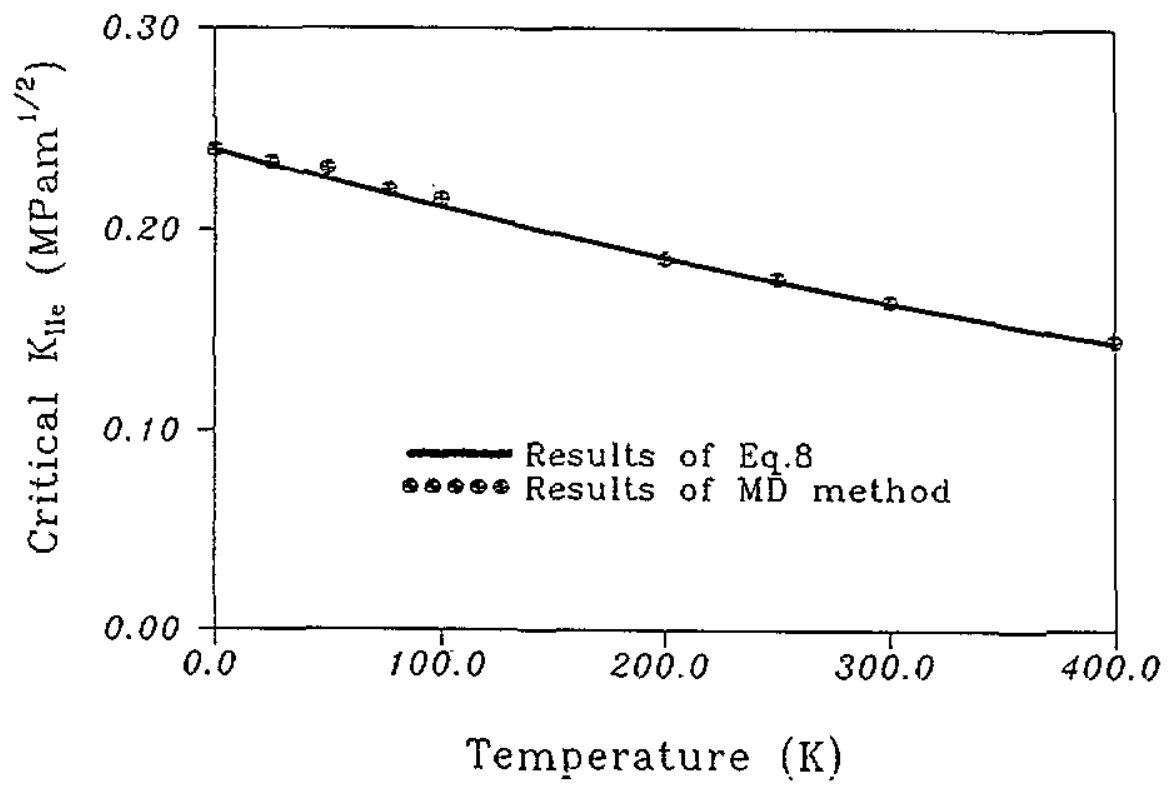

Figure 9. Comparison of the results of equation (8) with the molecular dynamics results for the critical stress intensity factor versus temperature.

is reduced by thermal activation. The dislocation velocity moving away from the crack tip only slightly increases with increases in temperature. At low temperatures, once the dislocation has been nucleated, it will move away from the crack tip quickly. But at high temperatures, when the dislocation has been nucleated, it does not move away from the crack tip at once, but hovers at the crack tip. The higher the temperature, the longer the incipient duration of dislocations. So at high temperature, The nucleation of dislocations does not mean the emission of dislocations. When the first dislocation reaches the displacement boundary, a pile-up of dislocations is set up. At high temperatures, the dislocation velocity becomes unsmooth, the dislocations may move back to crack tip locally and instantaneously. The stress obtained by the molecular dynamics method at $0 \mathrm{~K}$ is in good agreement with the anisotropic elasticity solution. As the temperature increases, the stress level near the crack tip increases slightly. Therefore the stress softening induced by the thermal activation does not exist in the present simulation. As $K_{\text {Ile }}$ is reduced by the thermal activation and the mean dislocation velocity from the crack tip is insensitive to the thermal activation, so the main effect of thermal activation on the ductility of crack tip is through the processes of dislocation nucleation and emission.

A simple model is used to evaluate the influence of thermal activation on the critical stress intensity factor. The exponential relation obtained between the critical stress intensity factor and temperature agrees well with our molecular dynamics results.

\section{Acknowledgments}

This project is supported by the National Natural Science Foundation of China. The authors would like to thank Professor F X Zhou for helpful discussions.

\section{References}

[1] Gilman J J 1960 J. Appl. Phys. 312208

[2] Hirsch P B, Roberts S G and Samuels J 1989 Proc. R. Soc. A 42125

[3] Rice J R 1992 J. Mech. Phys. Solid 40239

[4] Rice J R and Beltz G E 1994 J. Mech. Phys. Solid 42333

[5] Khantha M, Pope D P and Vitek V 1994 Phys. Rev. Lett. 73684

[6] Cheung K S, Argon A S and Yip S 1991 J. Appl. Phys. 69 2088

[7] Mullins M 1984 Acta Metall. 32381

[8] Kitagawa H and Nakatani A 1993 Proc. JSME A59-557 256

[9] Li J C M 1986 Scr. Metall. 201477

[10] Zhang Y W, Wang T C and Tang Q H 1995 J. Appl. Phys. 77 at press

[11] Finnis M W and Sinclair J E 1984 Phil. Mag. 5045

[12] Ackland G J, Tichy G, Vitek V and Finnis M W 1987 Phil. Mag. A56 735

[13] Woodcock L V 1971 Chem. Phys. Lett. 10257

[14] Nose S 1991 Computer Simulation in Materials Science ed $M$ Meyer and V Pontikis p 21

[15] Sih G C, Paris P C and Irwin G R $1965 \mathrm{~J}$, Fract. Mech. 1 189 